\title{
Perioperative Nursing Observation of Plasma Radiofrequency Ablation for Treatment of Lumbar Intervertebral Disc Herniation
}

\author{
Hui Sun ${ }^{\# *}$, Guo Tang", Dan Li, Lei Wang \\ Pain Department, First Medical Center of PLA General Hospital, Beijing 100853, China \\ ${ }^{\#}$ Hui Sun and Guo Tang both are the first authors and contributed equally to this work.
}

\begin{abstract}
[Abstract] Objective: To observe and analyze the perioperative nursing effect of plasma radiofrequency ablation for treatment of lumbar disc herniation. Methods: A total of 100 patients with lumbar intervertebral disc herniation received from October 2017 to October 2020 were selected and randomly divided into two groups with 50 cases in each group. The control group received the conventional nursing model and the experimental group received the comprehensive nursing model. The two groups of nursing care were compared on satisfaction, SDS and SAS scores before and after treatment, VAS score and JOA score before and after treatment, and treatment effect. Results: The nursing satisfaction of the experimental group, SDS and SAS scores before and after treatment, VAS score and JOA score before and after treatment, and the treatment effect were better than those of the control group. The difference was statistically significant $(\mathrm{P}<0.05)$. Conclusion: The comprehensive nursing model has a significant effect on the perioperative nursing of the treatment of lumbar intervertebral disc herniation by plasma radiofrequency ablation. More popularization and application will help improve the treatment effect.
\end{abstract}

Key words: Comprehensive nursing; Lumbar disc herniation; Plasma radiofrequency ablation

Publication date: May, 2021; Publication online: 31 May, 2021

*Corresponding author: Hui Sun, 1838716933@qq.com

\section{Introduction}

The number of patients with lumbar disc herniation is increasing year by year. The main clinical manifestations are low back pain, leg pain, etc., which greatly reduces the quality of life of patients and brings great troubles to their life and work. Traditional way of treatment is given priority to with conservative treatment, but the effect is not good, at present, the plasma radiofrequency ablation is an important surgical treatment of lumbar intervertebral disc protrusion method, but need long time of postoperative nursing rehabilitation exercise, as a result, the perioperative plasma radiofrequency ablation treatment of lumbar intervertebral disc protrusion care become a hot research topic in recent years $^{[1]}$. This study mainly focused on the effect of comprehensive nursing mode on perioperative nursing of plasma radiofrequency ablation in the treatment of lumbar disc herniation, and reported its nursing effect and safety as follows.

\section{Data and methods}

\subsection{General information}

A total of 100 patients with lumbar disc herniation admitted by our hospital from October 2017 to October 2020 were included in this study and randomly divided into two groups with 50 patients in each group. In the control group, the age was 26 to 70 years old, the mean age was $(49.63 \pm 6.24)$ years, the course of disease was 0.5 to 5.0 years, the mean course of disease was $(2.53 \pm 0.24)$ years, the lesion site was: 15 cases of L3-L4, 12 cases of L4-L5, 12 cases of L5-S1, 11 cases of L3-L4, L4-L5 double gap; There were 32 patients with simple protrusion and 18 patients with spinal stenosis. 
In the experimental group, the patients ranged from 25 to 66 years old, with an average age of (48.77 \pm 5.63$)$ years, course of disease 0.6 to 4.5 years, and average course of disease $(1.22 \pm 1.72)$ years. The lesion sites were as follows: 13 patients were L3-L4, 15 L4-L5, 9 L5-S1, and 13 patients were L3-L4, L4-L5 double gaps. There were 29 patients with simple protrusion and 21 patients with spinal stenosis, and there was no statistical significance in the basic data between the groups $(\mathrm{P}>0.05)$. Inclusion criteria :(1) meet the diagnostic criteria of "lumbar disc herniation"; (2) MRI showed lumbar disc herniation $<6 \mathrm{~mm}$; (3) Inclusive disc protrusion, annulus fibrous integrity; (4) The symptoms were not significantly improved or aggravated after conservative treatment; (5) Low back pain with unilateral lower limb radiating pain; (6) All patients signed the informed study agreement; (7) This study was approved by the ethics committee of the hospital. Exclusion criteria :(1) severe psychological and mental illness; (2) People with a history of drug allergy; (3) Patients with heart, liver, kidney and other serious organ dysfunction. (4) complicated with serious lumbar degenerative diseases, such as severe spinal stenosis, severe discotitis, lumbar spondylolisthesis, intervertebral disc calcification, etc.; (5) There was a history of interventional or surgical treatment at the lesion segment.

\subsection{Research methods}

\subsubsection{The control group}

Use routine care for patients, including routine anti-infective therapy, routine rehydration therapy, routine hormone therapy, and guide patients to get out of bed early. At the same time, for the treatment of patients with rehabilitation nursing guidance and training, including: (1) ask patients postoperative $3 \mathrm{~d}$, wearing the proper light activity around the waist, according to the rehabilitation, the guidance of a doctor doing moderate rehabilitation training, strength to strengthen gradually, including the direction of the core strength training, specific training, etc., according to the degree of each patient's condition, design the personalized training program for patients. (2) Low and medium frequency electrotherapy: the use of low and medium frequency electrotherapy equipment for rehabilitation after surgery, silicone electrode on both sides of the spine after the patient, 1 times $/ \mathrm{d}, 20 \mathrm{~min} /$ time, according to the specific extent of each patient's illness and personal patience, timely adjust the current frequency and intensity. (3) Psychological nursing for patients with pain tolerance ability is weak, give pain medication at the same time, the patients with psychological counseling ${ }^{[2]}$, timely communication with the patient, including detailed treatment of the communication with the patient relatives, increase the patient and family to know about lumbar intervertebral disc protrusion, help patients to build confidence. (4) timely help and guide the patient's family to help the patient turn over, maintain the axial turn over, ensure that the shoulder and hip are at the same level, can use a soft pillow to help the patient fixed, back and knee can be put two soft pillows, in order to reduce the symptoms of sciatic nerve compression.

\subsubsection{Group}

Line in the control group, on the basis of comprehensive nursing care model, including: (1) To form a comprehensive nursing working group, appointed head nurse group leader, then deputy team leader, appointed by the head nurse four head nurse to establish the comprehensive nursing measures, goals, arrangement and examination management system, and the team's members on what the deputy head of the group work, regulate the behavior of members in the group, in accordance with the assessment method of members on a regular basis for training and assessment work and improve the system of rewards and punishment; Other members of the group shall strictly abide by the system arrangements, actively promote the work, and participate in the work quality training and assessment activities on time. (2) Preoperative care: guide patients to conduct preoperative skin care, help patients to prepare the calf skin 1d before surgery, so as to paste the radio frequency instrument plate, and help patients to do routine preoperative examination in advance, including: blood routine, urine routine, electrocardiogram, chest CT, prothrombin time, etc ${ }^{[3]}$. Surgery patients with preoperative guidance position tolerance training, told the patient to keep for a long period of time prone position, with a soft pillow high abdomen, according to each patient tolerance ability to set the initial training time, then gradually increase the length, guarantee the training 3 times/d, at the same time, to guide the patients to cough effectively training, roll over method, how to safe to eat in bed and toilet training, etc ${ }^{[4]}$. If sleep is poor the night before, oral sleep-aid drugs can be given appropriately. (3) Intraoperative nursing: guiding patients in the intraoperative position, with pictures or models to help patients understand the intraoperative process, the possible situation, always observe the changes in the patient's psychological mood, timely communication with patients, help patients relax, improve the degree of cooperation. (4) 
Postoperative care: closely observe the patient's vital signs change, always pay attention to the puncture point, including bleeding, swelling, etc., at the same time, timely ask the patient lower limbs and waist pain, numbness, weakness and other symptoms of improvement; Accompanied by family members and careful activities to prevent falls; On the third day after the operation, the patient was given intravenous infusion of Aescinate Sodium and Glucose Injection to alleviate the edema of the vertebral space and the degree of postoperative pain. (5) Postural care: the patient was instructed to maintain supine position, sleep on a hard bed, and stay in bed for 3 days after surgery, so as to reduce the compression of the patient's lumbar spine, speed up the recovery of fibrous tissue, and avoid the occurrence of adverse reactions such as re-displacement and adhesion of intervertebral $\operatorname{disc}^{[5]}$. Once again, the patients were instructed to turn over the axial position during training, so as to reduce the incidence of spinal injury and postoperative complications. (6) Rehabilitation training to guide the patient remain recumbent posture, wear waist support strength, high leg training at the same time, 2 times/d, 20 min/time, training, instruct patients keep leg unbend, gradually raise the side of the lower extremities, until a sore, on both sides of the lower extremities interval training, according to patients' endurance level, gradually increase the training point of view, in order to promote blood circulation of patients with nerve root back quickly, reduce inflammation and tissue adhesions etc. Bad situation; On the third day after the operation, patients were instructed to start bedside training, gradually increase the intensity, keep the upper body upright, and always protect the waist circumference. Patients were encouraged to carry out wall supporting training as soon as possible, observe and correct the patients' bad training behaviors in time, and improve the training efficiency ${ }^{[6]}$. (7) All landowners diet nursing for patients with personalized menu, on what the patients' families, please, health education, strengthen the diet is given priority to with delicate, easy to digest food, to ensure nutritional balance, improve immunity, promote the exhaust and defecate, in high praise words on the compliance of patients, improve the enthusiasm of patients with healthy eating. (8) Rational drug use: through health education and other activities, to strengthen the patient's comprehensive understanding of lumbar disc herniation, improve health awareness, improve the patient's compliance, rational drug use, medication according to the doctor's advice. (9)
Emotional stabilization regulation: strengthen the observation of patients' emotional fluctuations, timely calm patients' nervous emotions, strengthen doctor-patient communication, reasonably and effectively help patients recover a positive attitude, and create a good nursing atmosphere. Through sharing videos, pictures and other forms, successful cases of surgery are listed to help patients establish good rehabilitation goals and confidence, improve the effect of nursing treatment, greatly accelerate patients' recovery time, improve the success rate of surgery, relieve patients' negative emotions, and improve patients' treatment compliance $^{[7]}$. Routine blood and erythrocyte sedimentation rate were checked before discharge.

\subsection{Observation indexes and evaluation criteria}

Nursing satisfaction, SDS and SAS scores before and after treatment, VAS scores and JOA scores before and after treatment, and treatment effect were observed and compared between the two groups.

\subsubsection{The triage accuracy rate and service satisfaction of} the two groups were observed and compared according to the satisfaction questionnaire filled out by patients (including service attitude, accuracy of expression, service quality, inquiry results and assisted examination, with 5 points for each item). Service satisfaction evaluation criteria:

- satisfaction was defined as patient satisfaction score $\geq 20$ points;

- Patients' satisfaction score $\geq 10$ points was considered as relatively satisfied;

- A patient satisfaction score of less than 10 was considered unsatisfactory;

- Total satisfaction $=$ satisfaction rate + comparative satisfaction rate.

\subsubsection{SDS and SAS scores}

20 topics were selected and self-tested by patients before and after treatment. The higher the score, the more significant the anxiety.

\subsection{Statistical methods}

SPSS 22.0 software was used for statistical analysis of the data. The enumeration data was expressed as rate $(\%), \chi^{2}$ test was used, and the measurement unit was (' $\mathrm{x} \pm \mathrm{s}$ ). $\mathrm{P}<0.05$ indicated a statistically significant difference.

\section{The result}

\subsection{Comparison of nursing satisfaction between the two groups}

The nursing satisfaction of the experimental group was 
significantly higher than that of the control group $(\mathrm{P}<0.05) . \quad$ See Table 1.

Table 1. Comparison of nursing satisfaction between the two groups (n,\%)

\begin{tabular}{cccccc}
\hline Group & The number of cases & Satisfaction $(\mathbf{n})$ & Satisfactory & Unsatisfactory & Satisfaction \\
\hline $\begin{array}{c}\text { Control group } \\
\text { experimental }\end{array}$ & 50 & $22(44.00)$ & $23(46.00)$ & $5(10.00)$ & $45(90.00)$ \\
group & 50 & $35(70.00)$ & $13(26.00)$ & $2(4.00)$ & $48(96.00)$ a \\
\hline
\end{tabular}

Note: there was significant difference between groups, $\mathrm{AP}<0.05$, and the difference was statistically significant.

3.2 Comparison of SDS and SAS scores before and The scores of SDS and SAS in the experimental group were after treatment between the two groups

better than those in the control group $(\mathrm{P}<0.05)$. See Table 2 .

Table 2. Comparison of SDS and SAS scores between the two groups before and after treatment (' $\mathrm{x} \pm \mathrm{s}$, points)

\begin{tabular}{|c|c|c|c|c|c|}
\hline \multirow{2}{*}{ Group } & \multirow{2}{*}{ The Number Of Cases } & \multicolumn{2}{|c|}{ SDS Score } & \multicolumn{2}{|c|}{ SAS Score } \\
\hline & & Before & After & Before & After \\
\hline Control group & 50 & $50.02 \pm 4.28$ & $48.56 \pm 3.65$ & $61.55 \pm 6.14$ & $55.33 \pm 5.46$ \\
\hline experimental group & 50 & $50.36 \pm 2.46$ & $42.36 \pm 4.38^{\mathrm{a}}$ & $61.74 \pm 6.22$ & $45.67 \pm 4.21^{\mathrm{a}}$ \\
\hline $\mathrm{t}$ & & 4.358 & 3.681 & 4.568 & 5.367 \\
\hline $\mathrm{P}$ & & $<0.05$ & $<0.05$ & $<0.05$ & $<0.05$ \\
\hline
\end{tabular}

Note: there was significant difference between groups, $\mathrm{AP}<0.05$, and the difference was statistically significant.

3.3 Comparison of VAS scores and JOA scores before and after treatment between the two groups VAS score and JOA score of the experimental group before and after treatment were significantly better than those of the control group $(\mathrm{P}<0.05)$. See Table 3.

Table 3. Comparison of VAS scores and JOA scores between the two groups before and after treatment (' $\mathrm{x} \pm \mathrm{s}, \mathrm{min})$

\begin{tabular}{cccccc}
\hline \multirow{2}{*}{ Group } & The Number & \multicolumn{2}{c}{ VAS score } & \multicolumn{2}{c}{ JOA score } \\
\cline { 3 - 6 } & of Cases & Before & After & Before & After \\
\hline Control group & 50 & $6.34 \pm 1.24$ & $2.56 \pm 0.44$ & $13.86 \pm 3.86$ & $22.65 \pm 2.38$ \\
experimental group & 50 & $6.27 \pm 1.67$ & $1.46 \pm 0.27^{\mathrm{a}}$ & $13.76 \pm 3.85$ & $19.54 \pm 2.65^{\mathrm{a}}$ \\
$\mathrm{t}$ & & 3.567 & 5.663 & 5.497 & 6.497 \\
$\mathrm{P}$ & & $<0.05$ & $<0.05$ & $<0.05$ & $<0.05$ \\
\hline
\end{tabular}

Note: there was significant difference between groups, $\mathrm{AP}<0.05$, and the difference was statistically significant.

2.4 Comparison of treatment effects between the two The effective rate of experimental group was significantly groups

higher than that of control group $(\mathrm{P}<0.05)$. See Table 4.

Table 4. Comparison of therapeutic effects between the two groups (n,\%)

\begin{tabular}{cccccc}
\hline Group & $\begin{array}{c}\text { The Number of } \\
\text { Cases }\end{array}$ & Excellent & Effective & Invalid & Total Effective Rate \\
\hline $\begin{array}{c}\text { Control group } \\
\text { experimental } \\
\text { group }\end{array}$ & 50 & $21(42.00)$ & $23(46.00)$ & $6(12.00)$ & $44(88.00)$ \\
\hline
\end{tabular}

Note: there was significant difference between groups, $\mathrm{AP}<0.05$, and the difference was statistically significant. 
cause of most of the patients with lumbocrural pain is caused by lumbar disc, due to reasons such as compression, wear, aging, patients with intervertebral disc fiber ring deformation, fracture, compression, cause the herniated, oppression horsetail nerve and nerve root, resulting in a related nerve pain and other symptoms ${ }^{[9]}$. The traditional treatment for patients with lumbar disc herniation is mainly conservative treatment. Although it has a certain effect, the effect is not good, and it is easy to have repeated attacks. The prognosis of patients is poor.

At present, in view of the main surgical treatment of lumbar disc prolapse is lumbar disc excision technique, the application of low-temperature plasma radiofrequency ablation is more and more widely, by minimally invasive treatment concept, using melting and heat setting, etc, intervertebral disc in patients with reduced pressure, make to intervertebral disc prominent retraction, try to reduce the patient's nerve damage ${ }^{[10]}$. Plasma radiofrequency ablation can minimize the chance of secondary injury during surgery ${ }^{[11]}$. By using the tension effect of annulus fibrosus and posterior longitudinal ligament, the prominent annulus fibrosus of patients can be automatically retracted, thus achieving the desired therapeutic effect. Low-temperature plasma radiofrequency ablation can also inactivate analgesic nerve fibers in patients, further reduce pain stimulation in patients, relieve pain in patients, and improve postoperative satisfaction in patients. Low temperature plasma radiofrequency ablation is safe, small trauma, high efficient, etc, can effectively improve the clinical symptoms in patients with lumbar disc prolapse, obvious effect, less adverse reaction, damage to the integrity of the fiber ring in patients with small ${ }^{[12]}$, greatly shorten the wound healing time, patients with accelerated rehabilitation patients, decrease the surgical trauma and improve the cure rate.

Comprehensive nursing is a new type of nursing in clinical nursing work mode, by judging the development conditions of the patients, and to develop a reasonable and targeted nursing measures, respectively in patients with postoperative early, middle and late period of three different, guiding patients with simple and effective exercise, gradually increase the exercise intensity, so as to speed up the patients recovered ${ }^{[13]}$. Application of comprehensive care, facilitate unified management of the patient's postoperative exercise, exercise can step by step to ensure patients, reducing excessive exercise, can effectively improve postoperative lower limb dysfunction in patients with lumbar disc prolapse, strengthen the patient's own immune system, shorten the patients' recovery time, improve the prognosis, greatly improve the patients quality of life, improve patient satisfaction ${ }^{[14,15]}$.

In this experiment, the total satisfaction of the experimental group was $96.000 \%$, higher than that of the control group $(90.00 \%)$. The effective rate of experimental group was $98.00 \%$, which was higher than that of control group (88.00\%). Moreover, SDS, SAS scores, VAS scores and JOA scores of the experimental group before and after treatment were significantly better than those of the control group, and the differences were statistically significant. It shows that the comprehensive nursing mode has a significant effect on perioperative nursing of plasma radiofrequency ablation for lumbar disc herniation.

In conclusion, the comprehensive nursing mode has a significant effect on the perioperative nursing of plasma radiofrequency ablation in the treatment of lumbar disc herniation, which is worthy of promotion and use.

\section{References}

[1] Zhang CF, Li XL, Peng WB, et al. A meta-analysis of traditional Chinese medicine, acupuncture, moxibustion and massage in the treatment of blood stasis type lumbar disc herniation [J]. Chinese Journal of Tissue Engineering Research, 2121,25(17):2781-2788.

[2] Liu HY, Zhang P, Yang JY, et al. Therapeutic effect of electroacupuncture Jiaji Point combined with Sanhan Huoxue Zhitong decoction and external application of traditional Chinese medicine in the treatment of lumbar intervertebral disc herniation and the effect on blood coagulation function and platelet $[\mathrm{J}]$. Thrombosis and Hemostasis,2020,26(05):756-758.

[3] Fang YH, Wang YL, Zou YC, et al. Clinical efficacy of lateral approach single-channel percutaneous foraminal surgery in the treatment of adjacent double-level lumbar disc herniation $[\mathrm{J}]$. Journal of Jilin Medical Sciences, 2019,41(09):2216-2218.

[4] Liu G, Tian Y, Shen XL, et al. Effects of zero-notch interbody fusion and nail plate system on postoperative curvature of single-level cervical disc herniation after anterior cervical discectomy and fusion [J]. Journal of Spinal Surgery,20,18(04):243-247+252.

[5] Lei J, Xiong M, Zhou S, et al. Clinical efficacy of posterior endoscopic cervical discectomy and anterior cervical discectomy with bone grafting and fusion for 
cervical disc herniation $[\mathrm{J}]$. Biological Orthopedic Materials and Clinical Research,2020,17(04):41-45.

[6] Jiang Y, Yuan L, Guo ZQ, et al. Transforaminal lumbar interbody fusion for the treatment of recurrent single-level lumbar disc herniation after percutaneous endoscopic lumbar discectomy [J]. Chinese Journal of Minimally Invasive Surgery, 201,21(01):41-46.

[7] Zhang LD, Yuan F, Chen HL, et al. Clinical efficacy of percutaneous foraminal Tessys and TLIF in the treatment of lumbar disc herniation and nerve root canal stenosis $[\mathrm{J}]$. Advances in Modern Biomedicine,2020,20(24):4696-4700.

[8] Sun WL, Su XY, Liu Y, et al. Effects of acupuncture and moxibustion combined with lumbar core muscle strength training on rehabilitation effect, quality of life and serum inflammatory factors in patients with lumbar disc herniation $[\mathrm{J}]$. Advances in Modern Biomedicine, 2020,20(22):4307-4310+4400.

[9] Lu M, Wang CH, Li M, et al. Intervertebral foraminal endoscopy in patients with human immunodeficiency virus infection with lumbar disc herniation: a non-randomized, controlled, 2-year follow-up clinical study [J]. Chinese Journal of Tissue Engineering Research, 2121,25(15):2375-2379.

[10] Du W, Ding Y, Cui HP, et al. Percutaneous endoscopic discectomy combined with platelet-rich plasma gel microsphere implantation in the treatment of lumbar disc herniation $[\mathrm{J}]$. Chinese Journal of Spine and Spinal Cord,2020,30(11):1001-1006.

[11] Xie ZJ, Liu QY. Comparison of the efficacy of percutaneous foraminal lumbar discectomy (PELD) and laminovened lumbar discectomy for lumbar disc herniation [J]. Smart Health,2020,6(30):48-49+58.

[12] Li DY, Teng HL, Lin CW, et al. Percutaneous endoscopic discectomy using visual bone chisel to remove part of the upper edge of the pedicle for treatment of extremely highly disassociative lumbar disc herniation $[\mathrm{J}]$. Journal of Spinal Surgery,20,18(05):310-314.

[13] Zhang HS, Tian BW, Chen L, et al. Effects of modified T's foraminal endoscopic technique on lumbar function and prognosis in patients with lumbar disc herniation and spinal stenosis $[\mathrm{J}]$. Jilin Journal of Traditional Chinese Medicine,2020,40(10):1339-1342.

[14] Wei ZQ, Zhang QF, Huang JH, et al. Effects of anterior cervical ROI-C implantation and conventional surgical treatment on JOA score and Cobb Angle [J]. Journal of Western Medicine,2020,32(08):1136-1141.

[15] Zhou SH, Wang LF, Zhong JH. The effect of percutaneous foraminal nursing on pain and quality of life in patients with lumbar intervertebral disc herniation $[\mathrm{J}]$. Journal of Clinical Rational Drug Use, 2020,13(23):141-143. 